\title{
Order of arrival promotes coexistence via spatial niche preemption by the weak competitor
}

\author{
Inês Fragata ${ }^{1}$, Raul Costa-Pereira ${ }^{2}$, Agnieszka Majer (Kiedrowicz) ${ }^{3}$, Oscar Godoy ${ }^{4}$, and \\ Sara Magalhães ${ }^{1}$ \\ ${ }^{1}$ Centre for Ecology Evolution and Environmental Changes \\ ${ }^{2}$ Universidade Estadual de Campinas Instituto de Biologia \\ ${ }^{3}$ Adam Mickiewicz University Faculty of Biology \\ ${ }^{4}$ Universidad de Cadiz Campus de Puerto Real
}

October 14, 2021

\begin{abstract}
Historical contingency, such as the order of species arrival, can modify competitive outcomes via niche modification or preemption. However how these mechanisms ultimately modify stabilising niche and average fitness differences remains largely unknown. By experimentally assembling two congeneric spider mite species feeding on tomato plants during two generations, we show that order of arrival interacts with species' competitive ability to determine competitive outcomes. Contrary to expectations, we did not observe that order of arrival cause priority effects. In fact, coexistence was predicted when the inferior competitor (Tetranychus urticae) arrived first. In that case, T. urticae colonized the preferred feeding stratum (leaves) of T. evansi leading to spatial niche preemption, which equalized fitness but also increased niche differences, driving community assembly to a close-to-neutrality scenario. Our study demonstrates how the spatial context of competitive interactions interact with species competitive ability to influence the effect of order of arrival on species coexistence.
\end{abstract}

\section{Hosted file}

MS_OrderOfArrival.pdf available at https://authorea.com/users/391521/articles/541652-orderof-arrival-promotes-coexistence-via-spatial-niche-preemption-by-the-weak-competitor 